\title{
COMPONENTS OF INVERTIBLE ELEMENTS IN QUOTIENT ALGEBRAS OF OPERATORS
}

\author{
BY \\ L. A. COBURN AND A. LEBOW( $\left.{ }^{1}\right)$
}

The purpose of this article is to present certain aspects of the theory of invertible elements in the various quotient algebras of the algebra, $\mathfrak{B}(\mathfrak{g})$, of all bounded operators on a Hilbert space $\mathfrak{S}$. In particular, the maximal connected subsets (components) of the set of left or right invertible elements in these quotient algebras are classified. A preliminary step is the geometric characterization of the operators in $\mathfrak{B}(\mathfrak{S})$ that are left or right invertible modulo an ideal of this algebra.

If $\mathfrak{S}$ is a Hilbert space and $\alpha$ is any infinite cardinal not larger than the dimension of $\mathfrak{B}$ then we denote by $\mathscr{I}_{\alpha}$ the set of operators in $\mathfrak{B}(\mathfrak{g})$ with the property that their ranges contain no closed subspace of dimension $\alpha$. It is part of the folklore that these sets $\mathscr{I}_{\alpha}$ are the only closed two-sided ideals of $\mathfrak{B}(\mathfrak{g})$.

When $\alpha$ is the smallest infinite cardinal $\boldsymbol{\aleph}_{0}$ it is known that $\mathscr{I}_{\alpha}$ is the ideal of compact operators. (Lemma 1 contains this result.) The transformations from one Banach space to another that have left or right inverses modulo the compact operators were characterized by Yood [5, p. 609]. It follows from the work of Atkinson [1] on Fredholm operators that there are infinitely many components of operators invertible modulo the compact operators on a Banach space $E$, if there is a subspace $F$ that is isomorphic to $E$ and $E / F$ is finite dimensional. The exact composition of these components was discovered by Cordes and Labrousse [4] for the case of a Hilbert space. In what follows we will also restrict our attention to Hilbert space but since we consider all ideals $\mathscr{I}_{\alpha}$, not just the ideal of compact operators, our results properly include those described above; at least in the case of a nonseparable Hilbert space.

The set of operators that are left invertible modulo $\mathscr{I}_{\alpha}$ will be denoted by $\mathscr{F}_{+}(\alpha)$. To be perfectly explicit:

$$
\mathscr{F}_{+}(\alpha)=\left\{F \in \mathfrak{B}(\mathfrak{g}): A F=I+J \text { for some } A \in \mathfrak{B}(\mathfrak{g}) \text { and } J \in \mathscr{I}_{\alpha}\right\} .
$$

In a similar way we define $\mathscr{F}_{-}(\alpha)$, the set of operators that are right invertible modulo $\mathscr{I}_{\alpha}$. Let $\mathscr{F}(\alpha)$ denote the union and $\mathscr{F}_{0}(\alpha)$ the intersection of $\mathscr{F}_{+}(\alpha)$ and $\mathscr{F}_{-}(\alpha)$. Lemma 2 is a characterization of operators in $\mathscr{F}_{+}(\alpha)$ which enables us to define an index on $\mathscr{F}(\alpha)$ in terms of the difference in nullity of an operator and its adjoint. Theorem 1 states that $\mathscr{F}_{0}(\alpha)$ is connected except when $\alpha=\boldsymbol{\aleph}_{0}$.

Received by the editors September 26, 1966.

(1) This work was supported in part by National Science Foundation Grants GP-5866 and GP-5436. 
To justify the title of this paper we refer to [2, p. 578] to conclude that the canonical map of $\mathfrak{B}(\mathfrak{H})$ into the quotient algebra $\mathfrak{B}(\mathfrak{S}) / \mathscr{I}_{\alpha}$ is a one to one correspondence between the components of $\mathscr{F}(\alpha)$ and the components of the set of left or right invertible elements of the quotient algebra.

We have been informed by H. O. Cordes that B. Gramsch has obtained results similar to our Lemma 1 in a paper to appear in Mathematische Annalen. Also, using his theory of " $R$-algebras," Cordes gives a generalization of our Lemma 2, characterizing semi-Fredholm elements, in a forthcoming paper [3].

The ideals $\mathscr{I}_{\alpha}$. An operator $T$ is said to be bounded below (above) on a subspace $\mathfrak{M}$, if there exists $\varepsilon>0$ such that $\|T x\| \geqq \varepsilon(<\varepsilon)$ for each unit vector $x$ in $\mathfrak{M}$. A subset $\mathscr{R} \subset \mathfrak{S}$ is said to be totally $\alpha$ bounded if for each $\varepsilon>0, \mathscr{R}$ can be covered by a collection of open $\varepsilon$-spheres of cardinality less than $\alpha$. We will require the following lemma which is well known in the special case of compact operators. Incidentally, this lemma can be made the basis of a proof that the $\mathscr{I}_{\alpha}$ are in fact closed two-sided ideals.

LEMMA 1. The following are equivalent

(i) The operator $T$ is in $\mathscr{I}_{\alpha}$.

(ii) Any closed subspace on which $T$ is bounded below is of dimension less than $\alpha$.

(iii) For each $\varepsilon>0$ there is a subspace $\mathfrak{M}$ of dimension less than $\alpha$ such that $T$ is bounded above by $\varepsilon$ on $\mathfrak{M}^{\perp}$.

(iv) The operator $T$ maps bounded sets into totally $\alpha$ bounded sets.

Proof. That (i) implies (ii) follows from the observation that if $T$ is bounded below on $\mathfrak{W}$ then $T(\mathfrak{B})$ has the same dimension as $\mathfrak{W}$. Now let $\mathfrak{R}$ be a closed subspace in the range of $T$. There is a subspace $\mathfrak{W}$ orthogonal to the null space of $T$ such that $T(\mathfrak{W})=\mathfrak{R}$. The closed graph theorem implies that $T$ is bounded below on $\mathfrak{W}$. This completes the proof that (i) and (ii) are equivalent.

Let $\left\{E_{\lambda}\right\}$ be the spectral resolution of the selfadjoint operator $H=T^{*} T$. Let $\mathfrak{W}_{\lambda}=E_{\lambda}(\mathfrak{S})$ so by computing the integral representation of $(H x, x)$ we have

$$
\|T x\|^{2}=(H x, x)=\int t d\left(E_{t} x, x\right) \geqq \lambda \int d\left(E_{t} x, x\right)=\lambda\|x\|^{2} \quad \text { for } x \text { in } \mathfrak{W}_{\lambda}^{\perp}
$$

and by a similar computation

$$
\|T x\|^{2} \leqq \lambda\|x\|^{2} \quad \text { for } x \text { in } \mathfrak{W}_{\lambda} .
$$

Now it follows from (ii) that $\operatorname{dim} \mathfrak{W}_{\lambda}^{\perp}<\alpha$ if $\lambda>0$ so that (iii) holds.

To show (iii) implies (iv) it suffices to show that $T(\mathfrak{S})$ is totally $\alpha$ bounded, where $\subseteq$ is the closed unit sphere of $\mathfrak{S}$. Let $\varepsilon>0$ be given and $\mathfrak{W}$ a closed subspace of dimension less than $\alpha$ such that

$$
\|T x\|<\varepsilon / 2 \quad \text { if } x \text { is in } \mathfrak{W}^{\perp} \text { and }\|x\| \leqq 1 .
$$


Since $\operatorname{dim} \mathfrak{W}<\alpha$ it is clear that $T(\subseteq \cap \mathfrak{W})$ can be covered by less than $\alpha$ open $(\varepsilon / 2)$-spheres. Given $x$ in $\mathfrak{S}, x=x_{1}+x_{2}$ with $x_{1}$ in $\mathfrak{W}^{\perp}$. Since

$$
\left\|T x-T x_{2}\right\|=\left\|T x_{1}\right\|<\varepsilon / 2,
$$

it follows from the triangle inequality that doubling the radii of the spheres in the cover of $T(\subseteq \cap \mathfrak{W})$ yields a cover of $T(S)$ of cardinality less than $\alpha$.

To complete the proof we show how (ii) follows from (iv). Suppose (ii) fails, then there is a subspace $\mathfrak{W}$ of dimension $\alpha$ such that

$$
\|T x\| \geqq \varepsilon\|x\| \quad \text { for } x \text { in } \mathfrak{W} .
$$

Let $\&$ be an orthonormal basis for $\mathfrak{W}$. For $x, y$ in $\&$

$$
\|T x-T y\|=\|T(x-y)\| \geqq \varepsilon \cdot 2^{1 / 2} \quad \text { if } x \neq y .
$$

Thus $T(\mathbb{E})$ cannot be covered by less than $\alpha$ open spheres of radius $\varepsilon \cdot 2^{-1 / 2}$.

The open semigroup $\mathscr{F}_{+}(\alpha)$. A special case of the result below is that an operator is left invertible modulo the compact operators if and only if it has a closed range and finite nullity [4].

Lemma 2. A necessary and sufficient condition that an operator $F$ is in $\mathscr{F}_{+}(\alpha)$ is that $F$ be bounded below on a subspace $\mathfrak{W}$ such that $\operatorname{dim} \mathfrak{W}^{\perp}<\alpha$ and $\mathfrak{W}^{\perp}$ contains the null space of $F$.

Proof. Let $A$ be an operator such that $A F=I+J$ with $J$ in $\mathscr{I}_{\alpha}$ and choose $\varepsilon$ such that $0<2\|A\| \varepsilon<1$. There is an orthonormal set $\&$ containing a basis for the null space of $F$ and maximal with respect to the property that $\|F x\|<\varepsilon$ for $x$ in $\mathbb{E}$; thus $F$ is bounded below on $\complement^{\perp}$. For distinct elements $x$ and $y$ in $\rightleftarrows$

$$
\begin{aligned}
\|J x-J y\| & \geqq\|x-y\|-\|x+J x\|-\|y+J y\| \\
& =2^{1 / 2}-\|A F x\|-\|A F y\| \\
& \geqq 2^{1 / 2}-2\|A\| \varepsilon>2^{1 / 2}-1 .
\end{aligned}
$$

Since $J(E)$ is totally $\alpha$ bounded by Lemma 1 , it follows that the cardinality of $\mathbb{E}$ is less than $\alpha$, which completes the proof of the necessity.

To prove the sufficiency let $P$ be the projection on $\mathfrak{W}^{\perp}$. The operator $F(I-P)$ is an isomorphism of $\mathfrak{W}$ onto $F(\mathfrak{W})$ so there exists an operator $A$ such that

$$
A F(I-P)=I-P \text {. }
$$

Since $P$ is in $\mathscr{I}_{\alpha}$ it follows that $F$ is in $\mathscr{F}_{+}(\alpha)$.

It is a consequence of Lemma 2 that the range of an operator in $\mathscr{F}_{+}(\alpha)$ is closed except for a space of dimension less than $\alpha$. Let $\mathfrak{N}$ be the orthogonal complement of $F(\mathfrak{W})$ in the closure of $F(\mathfrak{S})$. Thus

$$
F(\mathfrak{W}) \subset F(\mathfrak{H}) \subset F(\mathfrak{W}) \oplus \mathfrak{N} .
$$


Let $\mathfrak{D}$ be a dense subset of $\mathfrak{W}$ and $\mathfrak{E}$ be an orthonormal basis for $\mathfrak{N}$. If $e_{1}$ and $e_{2}$ are in $\mathbb{E}$ then there are vectors $d_{i}$ in $\mathscr{D}$ and $m_{\mathfrak{i}}$ in $\mathfrak{B}$ such that

$$
\left\|T\left(d_{i}+m_{i}\right)-e_{i}\right\|<2^{-1 / 2} .
$$

By the orthogonality of $T(\mathfrak{B})$ and $\mathfrak{N}$

$$
\left\|\left(T m_{1}-e_{1}\right)-\left(T m_{2}-e_{2}\right)\right\|^{2}=\left\|T\left(m_{1}-m_{2}\right)\right\|^{2}+\left\|e_{1}-e_{2}\right\|^{2} \geqq 2 .
$$

So it follows that $d_{1}$ and $d_{2}$ are distinct if $e_{1} \neq e_{2}$. Thus the cardinality of $\&$ is not greater than that of $\mathfrak{D}$. Thus, we have the following.

COROLlARY. If $F$ is in $\mathscr{F}_{+}(\alpha)$ then there are closed subspaces $\mathfrak{W}$ and $\mathfrak{R}$ such that

$$
\mathfrak{W} \oplus \mathfrak{R} \supset T(\mathfrak{H}) \supset \mathfrak{W} \quad \text { with } \operatorname{dim} \mathfrak{R}<\alpha .
$$

A dual to Lemma 2 for operators in $\mathscr{F}_{-}(\alpha)$ may be proved by noting that $T^{*}$ is bounded below on $T(\mathfrak{W})$ if $T$ is bounded below on $\mathfrak{W}$ and using well-known relations between an operator and its adjoint such as

$$
T^{*}\left(T(\mathfrak{W})^{\perp}\right) \subset \mathfrak{W}^{\perp} .
$$

The index and components. For any operator $F$ in $\mathscr{F}(\alpha)$ either $F$ or $F^{*}$ has nullity less than $\alpha$ by Lemma 2 . We denote the nullity of $F$ by $n(F)$ and $n\left(F^{*}\right)$ is called the defect of $F$. (Recall that the null space of $F^{*}$ is $F(\mathfrak{S})^{\perp}$.) The index of $F$ is defined to be

$$
i_{\alpha}(F)=n\left(F^{*}\right)-n(F)
$$

except that if $\alpha$ is uncountable and both $n(F)$ and $n\left(F^{*}\right)$ are less than $\alpha$ we set

$$
i_{\alpha}(F)=0, \text { by definition. }
$$

Theorem 1 is the main justification for this definition. Consideration of the special case of operators of the form $I+J$ may be helpful in understanding why index 0 is defined as it is. It follows from the classical Riesz-Schauder theory that $n(I+J)$ and $n\left(I+J^{*}\right)$ are finite and equal if $J$ is compact. If it is known only that $J$ is in $\mathscr{I}_{\alpha}$, it is not always true that $n(I+J)=n\left(I+J^{*}\right)$ even though both are less than $\alpha$. However, it is clear that $I+J$ is in the component of $I$. Thus if the index $n\left(F^{*}\right)-n(F)$ is to be constant on components, cardinals less than $\alpha$ must be ignored when $\alpha \neq \aleph_{0}$.

THEOREM 1. Two operators are in the same component of $\mathscr{F}(\alpha)$ if and only if they have the same index.

Proof. By the continuity of the adjoint operation it suffices to consider an operator $F$ in $\mathscr{F}(\alpha)$ with $n(F) \leqq n\left(F^{*}\right)$ so that $F$ has nonnegative index and $n(F)<\alpha$. Consider the polar factorization of $F=W H$ where $H$ is the positive square root of $F^{*} F$ and $W$ is the partial isometry from the orthogonal complement of the null space of $F$ onto the closure of its range. Let $W^{\prime}$ be any partial isometry from the 
null space of $F$ into $F(\mathfrak{g})^{\perp}$. Now $V=W+W^{\prime}$ is an isometry with the same index as $F$. To show that $F$ and $V$ are in the same component of $\mathscr{F}(\alpha)$ let us define an arc by

$$
\phi(t)=V[(1-t) H+t I] .
$$

For any $t \neq 0, \phi(t)$ is left invertible and thus in $\mathscr{F}(\alpha)$ and $\phi(0)=F$. We now need to show that two isometries of the same index are in the same component. Suppose $V_{1}$ and $V_{2}$ are isometries with the same defect. Let $W$ be a partial isometry mapping $V_{1}(\mathfrak{G})^{\perp}$ onto $V_{2}(\mathfrak{g})^{\perp}$ so $U=V_{2} V_{1}^{*}+W$ is unitary and $U V_{1}=V_{2}$. It follows from the connectedness of the unitary group that $V_{1}$ and $V_{2}$ are in the same component of $\mathscr{F}(\alpha)$. To complete this half we need only consider the case of an isometry $V$ of index 0 and defect less than $\alpha$, an uncountable cardinal. We have just shown that $V$ is in the same component as $I \oplus S$ where $S$ is a shift of multiplicity $n\left(V^{*}\right)<\alpha$. Since $\alpha$ is uncountable it follows that the range of $0 \oplus S$ has dimension $n\left(V^{*}\right) \cdot \mathcal{N}_{0}<\alpha$ and so $0 \oplus S$ is in $\mathscr{I}_{\alpha}$ and thus $I \oplus S$ can be connected to $I$ by an $\operatorname{arc}$ in $\mathscr{F}_{0}(\alpha)$.

To prove the converse it is sufficient to show that the sets of operators with constant index are open. We will accomplish this in two steps. First it will be shown that every isomorphism (with closed range) has a neighborhood which consists of isomorphisms of the same defect. Second it will be shown that the addition of an operator in $\mathscr{I}_{\alpha}$ to an isomorphism does not change its index. From Lemmas 1 and 2 it is easy to see that for any operator $F$ in $\mathscr{F}(\alpha)$ with nonnegative index there exists $J$ in $\mathscr{I}_{\alpha}$ such that $F+J$ is an isomorphism. The translate by $-J$ of a neighborhood of $F+J$ with constant index is a neighborhood of $F$ with constant index.

First step. If $A$ is an isomorphism then there is an operator $B$ such that $B A=I$. Let $\|C\|<\|B\|^{-1}$ then $\|C B\|<1$ so that $(I+C B)^{-1}$ exists. To show that $B(I+C B)^{-1}$ is a left inverse for $A+C$ we recall that

Thus we have

$$
(I+C B)^{-1}=I-C(I+B C)^{-1} B .
$$

$$
\begin{aligned}
B(I+C B)^{-1}(A+C) & =B\left[I-C(I+B C)^{-1} B\right](A+C) \\
& =B(A+C)-B C(I+B C)^{-1} B A-B C(I+B C)^{-1} B C \\
& =I+B C-B C(I+B C)^{-1}(I+B C)=I .
\end{aligned}
$$

Moreover

$$
(A+C) B(I+C B)^{-1}=(A B+C B A B)(I+C B)^{-1}=(I+C B) A B(I+C B)^{-1} .
$$

Thus $A B$ and $(A+C) B(I+C B)^{-1}$ are similar and since these idempotents are projections on the ranges of $A$ and $A+C$, respectively, it follows that $A$ and $A+C$ have the same defect.

Second step. Suppose $A$ is an isomorphism and $J$ is in $\mathscr{J}_{\alpha}$. We wish to show that

$$
i_{\alpha}(A)=i_{\alpha}(A+J) \text {. }
$$


Let $B$ be an operator with null space $N(B)=A(\mathfrak{S})^{\perp}$ such that $B A=I$. As in the corollary to Lemma 2 , the closure of $(A+J) \mathfrak{S}$ is of the form $\mathfrak{W} \oplus \mathfrak{N}$ with

$$
\mathfrak{W} \oplus \mathfrak{R} \supset(A+J) \mathfrak{H} \supset \mathfrak{W},
$$

$\mathfrak{W}$ closed, and $\operatorname{dim} \mathfrak{\Re}<\alpha$.

Let

$$
\begin{aligned}
\mathfrak{X}_{0} & =N(B) \cap \mathfrak{W}, \\
\mathfrak{W}_{1} & =\mathfrak{W} \cap \mathfrak{X}_{0}^{\perp} \\
\mathfrak{X}_{1} & =N(B) \cap \mathfrak{R} \cap(A+J) \mathfrak{H}_{3} \\
\mathfrak{X}_{2} & =\mathfrak{N} \cap \mathfrak{X}_{1}^{\perp} \\
\mathfrak{X}_{3} & =N(B) \cap(\mathfrak{W} \oplus \mathfrak{P})^{\perp}, \\
\mathfrak{X}_{4} & =\left(\mathfrak{W}_{1} \oplus \mathfrak{X}_{0} \oplus \mathfrak{X}_{1} \oplus \mathfrak{X}_{2} \oplus \mathfrak{X}_{3}\right)^{\perp} .
\end{aligned}
$$

It should be clear that

$$
\begin{aligned}
N(B) & =\mathfrak{X}_{0} \oplus \overline{\mathfrak{X}}_{1} \oplus \mathfrak{X}_{3}, \\
{\left[(A+J) \mathfrak{S}^{\perp}\right.} & =\mathfrak{X}_{2} \oplus \mathfrak{X}_{3} \oplus \mathfrak{X}_{4} .
\end{aligned}
$$

If $\alpha_{i}$ denotes the dimension of $\mathfrak{X}_{i}$ it follows that

and

$$
n[B(A+J)]=n(A+J)+\alpha_{0}+\alpha_{1}
$$

$$
n\left[\left(A^{*}+J^{*}\right) B^{*}\right]=\alpha_{2}+\alpha_{4} .
$$

Since $B(A+J)=I+B J$ is an operator of index 0 we have

$$
\begin{aligned}
i_{\alpha}(A+J) & =\left(\alpha_{2}+\alpha_{3}+\alpha_{4}\right)-\left(\alpha_{2}+\alpha_{4}-\alpha_{0}-\alpha_{1}\right) \\
& =\alpha_{0}+\alpha_{1}+\alpha_{3}=n(B)=i_{\alpha}(A)
\end{aligned}
$$

whether or not we are in the situation of ignoring cardinals less than $\alpha$.

CoRollaRY 1. If $F$ is in $\mathscr{F}(\alpha)$ and $J$ in $\mathscr{I}_{\alpha}$, then $i_{\alpha}(F+J)=i_{\alpha}(F)$.

CoROllaRY 2. If $F$ and $G$ are in $\mathscr{F}_{+}(\alpha)\left(\mathscr{F}_{-}(\alpha)\right)$, then $i_{\alpha}(F G)=i_{\alpha}(F)+i_{\alpha}(G)$.

It follows immediately from Theorem 1 that $\mathscr{F}_{0}(\alpha)$ is connected for $\alpha>\boldsymbol{\aleph}_{0}$ so that by $[2$, p. 578] we have:

COROllaRY 3. The group of invertible elements in $\mathfrak{B}\left(\mathfrak{H}_{\alpha}\right) / \mathscr{I}_{\alpha}$ is connected for all $\alpha>\boldsymbol{N}_{0}$.

It is easy to see from Theorem 1 that $i_{\alpha}$ is an index, in the sense of [2], on $\mathscr{F}_{+}(\alpha)$ (and on $\mathscr{F}_{-}(\alpha)$ ). For $\alpha>\aleph_{0}$ the range of $i_{\alpha}$ on $\mathscr{F}_{+}(\alpha)$ is the semigroup of 0 and all cardinals between $\alpha$ and the dimension of the underlying Hilbert space.

\section{REFERENCES}

1. F. V. Atkinson, The normal solubility of linear equations in normed spaces, Mat. Sb. 28 (70) (1951), 3-14.

2. L. A. Coburn and A. Lebow, Algebraic theory of Fredholm operators, J. Math. Mech. 15 (1966), 577-584. 
3. H. O. Cordes, On a generalized Fredholm theory, J. Reine Angew. Math. (to appear).

4. H. O. Cordes and J. P. Labrousse, The invariance of the index in the metric space of closed operators, J. Math. Mech. 12 (1963), 693-720.

5. B. Yood, Properties of linear transformation invariant under addition of a completely continuous transformation, Duke Math. J. 18 (1951), 599-612.

YeSHIVA UNIVERSITY,

NEW YoRK, NeW YoRK

UNIVERSITY OF CALIFORNIA,

IrVINe, CALIFornia 